\title{
Crystal Structure of the Apo and the ADP-Bound Form of Choline Kinase from Plasmodium falciparum
}

\author{
Archimede Torretta ${ }^{1}$, Luisa Carlota Lopez-Cara ${ }^{2}$ - and Emilio Parisini ${ }^{1,3, *}$ \\ 1 Center for Nano Science and Technology @PoliMi, Istituto Italiano di Tecnologia, Via Pascoli 70/3, \\ 20133 Milano, Italy; archimede.torretta@iit.it \\ 2 Department of Pharmaceutical and Organic Chemistry, Faculty of Pharmacy, Campus of Cartuja, \\ 18071 Granada, Spain; lcarlotalopez@ugr.es \\ 3 Latvian Institute of Organic Synthesis, Aizkraukles 21, LV-1006 Riga, Latvia \\ * Correspondence: emilio.parisini@osi.lv
}

Received: 12 June 2020; Accepted: 11 July 2020; Published: 14 July 2020

\begin{abstract}
Among the malaria-causing parasites, the deadliest is Plasmodium falciparum, which accounts for the majority of the fatalities. As the infection progresses inside erythrocytes, major cellular and metabolic changes take place. For its own growth, the parasite relies on the accumulation of phospholipids, which are essential for membrane synthesis. Within the Kennedy pathway, the P. falciparum choline kinase (PfChoK) has a central role in the biosynthesis of phosphatidylcholine and its selective inhibition leads to the parasite arrest and eradication. Here, we report the crystal structure of the apo and the ADP-bound form of choline kinase from Plasmodium falciparum at 2.0 and $2.2 \AA$ resolution, respectively. These new structural data will facilitate the implementation of effective structure-based drug development strategies against PfChoK in the fight against malaria.
\end{abstract}

Keywords: choline kinase; Plasmodium falciparum; malaria; ADP; crystal structure

\section{Introduction}

Malaria remains a major public health concern, especially in the African region, where most of the cases are reported. According to the latest "World Health Organization Malaria Report" [1], between 206 and 258 million cases were reported worldwide in 2018, resulting in an estimated death toll of $405,000,67 \%$ of which occurred amongst children aged under 5 , the most vulnerable population to the disease. Malaria is caused by the blood-parasites protozoa of the Plasmodium genus. Amongst the four human malaria parasites (P. falciparum, Plasmodium vivax, Plasmodium ovale, and Plasmodium malariae) [2], Plasmodium falciparum is the deadliest and is accountable for the majority of the estimated cases [1].

Unfortunately, while conventional antimalarial remedies comprise artemisinin-based combination therapies, chloroquine, sulfadoxine-pyrimethamine, and amodiaquine [3], resistance to these conventional therapies has been reported worldwide [4,5]. Hence, there is an urgent need for new antimalarial drugs to eradicate chemotherapy-resistant strains.

During their 48-hour long asexual intra-erythrocytic maturation cycle, P. falciparum merozoites go through different developmental stages (ring, trophozoite, and schizont state), which lead to 36 new daughter parasite cells [6]. Each of these cells is capable of beginning a new intra-erythrocytic life cycle, thus fostering the spread of the disease and causing the characteristic pathological symptoms of malaria (headache, fatigue, abdominal pain, fever, chills, perspiration, vomiting, metabolic acidosis, anemia, hypoglycemia, renal failure, and pulmonary edema) $[3,4,7]$. 
The intra-erythrocytic growth of the parasite is accompanied by substantial biochemical and biophysical changes in the host cell membrane. These changes are required in order to internalize large amounts of nutrients and to dispose of the waste products of the parasite metabolism [8,9].

One of the most striking consequences of the blood-stage parasitization is the dramatic increase of the phospholipid content, especially phosphatidylcholine (PC) and phosphatidylethanolamine $(\mathrm{PE})$, inside the infected erythrocyte, which can reach up to five times its physiological level; this is a necessary requirement for the parasite membrane biosynthesis and, therefore, for its growth and replication [2,10-14].

Interestingly, erythrocytes are enucleated cells that lack the enzymatic machinery for the de novo synthesis of phospholipids. Moreover, P. falciparum plasma membrane is mainly composed by PC and $\mathrm{PE}$, which represent $40-50 \%$ and $35-40 \%$ of the total phospholipid content, respectively [15]. Hence, targeting the parasite lipid metabolism for pharmacological action constitutes an effective way of dealing with the spreading of the disease and with conventional therapy-resistant strains [2,10-13].

Targeting the PC biosynthetic pathways by designing choline analogues has been shown to be lethal for the parasite in in vitro experiments [16-19]. In P. falciparum, PC biosynthesis is operated by two distinct metabolic pathways: the CDP-choline or Kennedy pathway and the serine decarboxylation-phosphoethanolamine methylation pathway $[20,21]$. The former is responsible for $89 \%$ of PC synthesis, while the latter accounts for the remaining $11 \%$ [22]. The Kennedy pathway consists of a three-step enzymatic cascade, whereby choline is transformed into PC through the action of choline kinase (ChoK), СTP:phosphocholine cytidylyltransferase, and choline/ethanolamine phosphotransferase [23].

ChoK has been proved to be a cytosolic marker for human erythrocyte-infection by P. falciparum [16]; ChoK selective inhibition causes the asexual intra-erythrocytic cycle to arrest and results in the death of the parasite [24-28]. Despite an extensive biochemical characterization of P. falciparum choline kinase (PfChoK) [29-31] and the elucidation of its underlying ping-pong catalytic mechanism [25], reliable and complete structural data that can guide the design of new and more potent inhibitors have so far been partially elusive. Here, we present the crystal structure of PfChoK (EC 2.7.1.32) in its apo and in its ADP-bound conformation.

\section{Materials and Methods}

\subsection{Cloning, Protein Expression, and Purification}

A truncated form of PfChoK ( $\triangle 79-440)$ featuring an $\mathrm{N}$-terminal $6 x$ His tag and cloned into a pET-28a vector (Invitrogen, Carlsbad, CA, USA) was purchased from Genescript (Piscataway, NJ, USA) and used to transform Escherichia coli BL21 (DE3) Star cells (Invitrogen, Carlsbad, CA, USA). Cells were then cultured in Luria-Bertani (LB) medium until $\mathrm{OD}_{600}=0.6$, when protein expression was induced using $1 \mathrm{mM}$ isopropyl $\beta$-D-1-thiogalactopyranoside (IPTG). The culture was incubated overnight at $180 \mathrm{rpm}$ and $20^{\circ} \mathrm{C}$. Cells were then pelleted by centrifugation at $10,000 \mathrm{rpm}$, resuspended in $50 \mathrm{mM}$ Tris/ $\mathrm{HCl} \mathrm{pH} \mathrm{7.5,} 500 \mathrm{mM} \mathrm{NaCl}, 0.2 \mathrm{mM}$ phenylmethylsulphonyl fluoride (PMSF, Sigma Aldrich, St. Luis, MO, USA), DNase (Sigma Aldrich, St. Luis, MO, USA), $0.5 \mathrm{mM} \beta$-mercaptoethanol (Sigma Aldrich, St. Luis, MO, USA), and sonicated. The soluble fraction was separated from the cell debris by centrifugation at $15,000 \mathrm{rpm}$ and $4{ }^{\circ} \mathrm{C}$.

The PfChoK enzyme was isolated following a two-step purification protocol. In a first affinity chromatography step, the soluble fraction was incubated with Ni-NTA agarose beads (Qiagen, Venlo, The Netherlands) for $45 \mathrm{~min}$. After washing the column extensively with a $50 \mathrm{mM}$ Tris/ $\mathrm{HCl} \mathrm{pH} 7.5$, $300 \mathrm{mM} \mathrm{NaCl}, 40 \mathrm{mM}$ imidazole buffer solution, the His-tagged PfChoK was eluted in $50 \mathrm{mM}$ Tris/ $\mathrm{HCl}$ $\mathrm{pH} 7.5,300 \mathrm{mM} \mathrm{NaCl}$, and $400 \mathrm{mM}$ imidazole. The protein was then further purified by size-exclusion chromatography using a HiPrep 26/60 Sephacryl 100 HR column (GE Healthcare, Little Chalfont, Buckinghamshire, $\mathrm{UK}$ ) and a $20 \mathrm{mM}$ Tris/ $\mathrm{HCl} \mathrm{pH} \mathrm{7.5,} 150 \mathrm{mM} \mathrm{NaCl}$ running buffer. The final protein production yield was in the order of $2.5 \mathrm{mg}$ of recombinant protein per liter of bacterial culture. 


\subsection{Crystallization}

Crystals of the apo and of the ADP-bound PfChoK enzyme were obtained by the hanging drop vapor diffusion method using VDXm 24-well plates with siliconized glass circle slides (Hampton Research, Aliso Viejo, California, USA) and screening around the crystallization conditions reported in the PDB for the incomplete crystal structure of phosphocholine- and ADP-bound PfChoK (3FI8) (unpublished data). In each experiment, $1 \mu \mathrm{L}$ of a $10 \mathrm{mg} / \mathrm{mL}$ PfChoK sample solution was mixed with an equal volume of crystallization buffer and the droplet was left equilibrating against a $500 \mu \mathrm{L}$ reservoir at room temperature, resulting in crystals of suitable dimensions within one week. Crystals of the apoenzyme grew in a $16 \%(v / v)$ polyethylene glycol (PEG) $8000,0.2 \mathrm{M} \mathrm{NaCl}, 0.1 \mathrm{M}$ HEPES pH 7.5, $2 \mathrm{mM}$ Tris(2-carboxyethyl)phosphine (TCEP), and $4 \mathrm{mM} \mathrm{MgCl}_{2}$ crystallization buffer. Likewise, crystals of the ADP-bound form of the enzyme were obtained in $16 \%(v / v)$ polyethylene glycol (PEG) 4000, 0.2 M NaCl, 0.1 M HEPES pH 7.5, 2 mM Tris(2-carboxyethyl)phosphine (TCEP), $4 \mathrm{mM} \mathrm{MgCl}{ }_{2}$, and $2 \mathrm{mM}$ ADP. Crystals of the apo and of the ADP-bound enzyme were then flash-frozen in $25 \%(v / v)$ glycerol and in $25 \%(v / v)$ ethylene glycol, respectively, for X-ray diffraction experiments.

\subsection{X-Ray Diffraction Data Acquisition, Structure Solution, and Refinement}

A $2.0 \AA$ resolution dataset and a $2.2 \AA$ dataset were collected for the apo and for the ADP-bound enzyme, respectively, at the Swiss Light Source (Paul Scherrer Institute, Villigen, Switzerland) using a $\lambda=1.000 \AA$ (beamline X06DA-PXIII). Diffraction images were processed using iMosflm [32] and scaled with SCALA in the CCP4 program suite (Oxford, UK) [33]. Molecular replacement and model refinement were done using Phenix (Berkeley, CA, USA) [34] and the incomplete crystal structure of phosphocholine- and ADP-bound PfChoK as the search and starting model (PDB code: 3FI8). During refinements, manual adjustments to the model were performed with Coot [35]. Data collection and refinement statistics for the two crystal structures are shown in Table 1. Molecular graphics and analyses were done using UCSF Chimera (University of San Francisco, San Francisco, CA, USA) [36] and Pymol (Schrödinger Inc., New York, NY, USA) [37].

Table 1. Data collection and refinement statistics.

\begin{tabular}{|c|c|c|}
\hline Crystallographic Table & PfChoK Apo Form & PfChoK ADP-Bound Form \\
\hline Protein Data Bank ID & $6 \mathrm{YXS}$ & $6 \mathrm{YXT}$ \\
\hline Space group & $\mathrm{P} 2_{1} 2_{1} 2_{1}$ & $\mathrm{P} 2_{1} 2_{1} 2_{1}$ \\
\hline \multicolumn{3}{|l|}{ Cell dimensions } \\
\hline$a, b, c(\AA)$ & $66.41,68.82,104.80$ & $66.45,67.99,105.11$ \\
\hline$\alpha, \beta, \gamma\left({ }^{\circ}\right)$ & $90,90,90$ & $90,90,90$ \\
\hline Wavelength (Å) & 1.00 & 1.00 \\
\hline Resolution (̊̊) & $57.53-2.00(2.11-2.00)$ & $68.00-2.20(2.32-2.20)$ \\
\hline Total reflections & $263,600(40,520)$ & $125,859(17,449)$ \\
\hline Unique reflections & $33,217(4770)$ & $24,873(3571)$ \\
\hline Multiplicity & $7.9(8.5)$ & $5.1(4.9)$ \\
\hline Completeness (\%) & $100.0(100.0)$ & $99.90(100.0)$ \\
\hline $\mathbf{R}_{\text {merge }}$ & $0.203(1.736)$ & $0.146(1.593)$ \\
\hline Mean I/sigma(I) & $8.00(1.3)$ & $6.5(1.00)$ \\
\hline Wilson B-factor $\left(\AA^{2}\right)$ & 24.5 & 37.1 \\
\hline $\mathrm{CC} 1 / 2$ & $0.995(0.429)$ & $0.994(0.486)$ \\
\hline Reflections used in refinement & $33,149(3242)$ & $24,706(2339)$ \\
\hline Reflections used for $R_{\text {free }}$ & 1739 (159) & $1230(114)$ \\
\hline
\end{tabular}


Table 1. Cont.

\begin{tabular}{|c|c|c|}
\hline Crystallographic Table & PfChoK Apo Form & PfChoK ADP-Bound Form \\
\hline $\mathbf{R}_{\text {work }}$ & $0.2063(0.3247)$ & $0.2305(0.4671)$ \\
\hline $\mathbf{R}_{\text {free }}$ & $0.2385(0.3812)$ & $0.2553(0.4983)$ \\
\hline Total number of non-H atoms & 3277 & 3165 \\
\hline protein & 3020 & 3028 \\
\hline ligands & 53 & 41 \\
\hline solvent & 204 & 96 \\
\hline Protein residues & 359 & 359 \\
\hline RMS bonds (Å) & 0.003 & 0.002 \\
\hline RMS angles $\left({ }^{\circ}\right)$ & 0.53 & 0.66 \\
\hline \multicolumn{3}{|l|}{ Ramachandran plot } \\
\hline Favored/allowed/outliers (\%) & $96.36 / 2.80 / 0.00$ & $92.16 / 5.60 / 2.24$ \\
\hline B-factor (average) $\left(\AA^{2}\right)$ & 38.25 & 57.15 \\
\hline protein & 37.84 & 57.32 \\
\hline ligands & 49.42 & 50.23 \\
\hline solvent & 41.36 & 54.66 \\
\hline
\end{tabular}

Statistics for the highest-resolution shell are shown in parentheses.

\section{Results and Discussion}

We produced a 361 amino acid-long truncated form of the enzyme lacking the first $79 \mathrm{~N}$-terminal residues. As shown in previous structural studies on the nematode Caenorhabditis Elegans (PDB code: 1NW1) [38] and human (PDB code: 2CKO) [39] variants (ChoKA-2 and hChoK $\alpha 1$, respectively), this N-terminal domain features a non-conserved region (Figure 1) that plays no role in catalysis. The structure of the PfChoK apoenzyme was solved by molecular replacement using the incomplete crystal structure of phosphocholine- and ADP-bound PfChoK available in the PDB (3FI8) (paper not published) as search model. Each missing loops and side chain in the starting model were manually assigned to fill all the available electron density and the model was refined at $2.00 \AA$ resolution. Then, the coordinates of the apoenzyme were used as starting model for solving the ADP-bound structure of the enzyme, which was refined at $2.20 \AA$ resolution.

Like other choline kinase eukaryotic enzymes, the structure of both the PfChoK apoenzyme and its ADP-bound form feature the typical globular and bilobate eukaryotic protein kinase fold (Figure 2), consisting of a 184-residue long N-terminal domain and a 245-residue long C-terminal domain that are connected by a short linker region.

The N-terminal domain begins with one $\alpha$ helix and two $3_{10}$ helices $(\alpha 1, \eta 1$, and $\eta 2$, Figure 3 , blue). Helix $\eta 2$ is followed by a five-stranded twisted antiparallel $\beta$ sheet (Figure 3, light sea green), a highly conserved structural motif among eukaryotic protein kinases, especially strands $A$ and $B$, which contain the ATP-binding motif. The $\alpha 2$ helix is inserted between strands B and C (Figure 3, purple), while helices $\eta 3$ and $\alpha 3$ are inserted between strands $C$ and D (Figure 3 , magenta). Helices $\alpha 2$ and $\eta 3$ are not found in ChoKA-2 and hChoK $\alpha 1$ and constitute a unique feature of PfChoK, while helix $\alpha 3$ is shorter than the corresponding helices in ChoKA-2 and hChoK $\alpha 1$. Interestingly, whereas helix $\alpha 3$ mediates dimer formation, both in the ChoKA-2 and in the hChoK $\alpha 1$ crystal structure, the structure of PfChoK is monomeric. Consistent with this observation, analytical size-exclusion chromatography analysis shows that the oligomerization state of PfChoK in solution is also monomeric (Supplementary Materials Figure S1). 


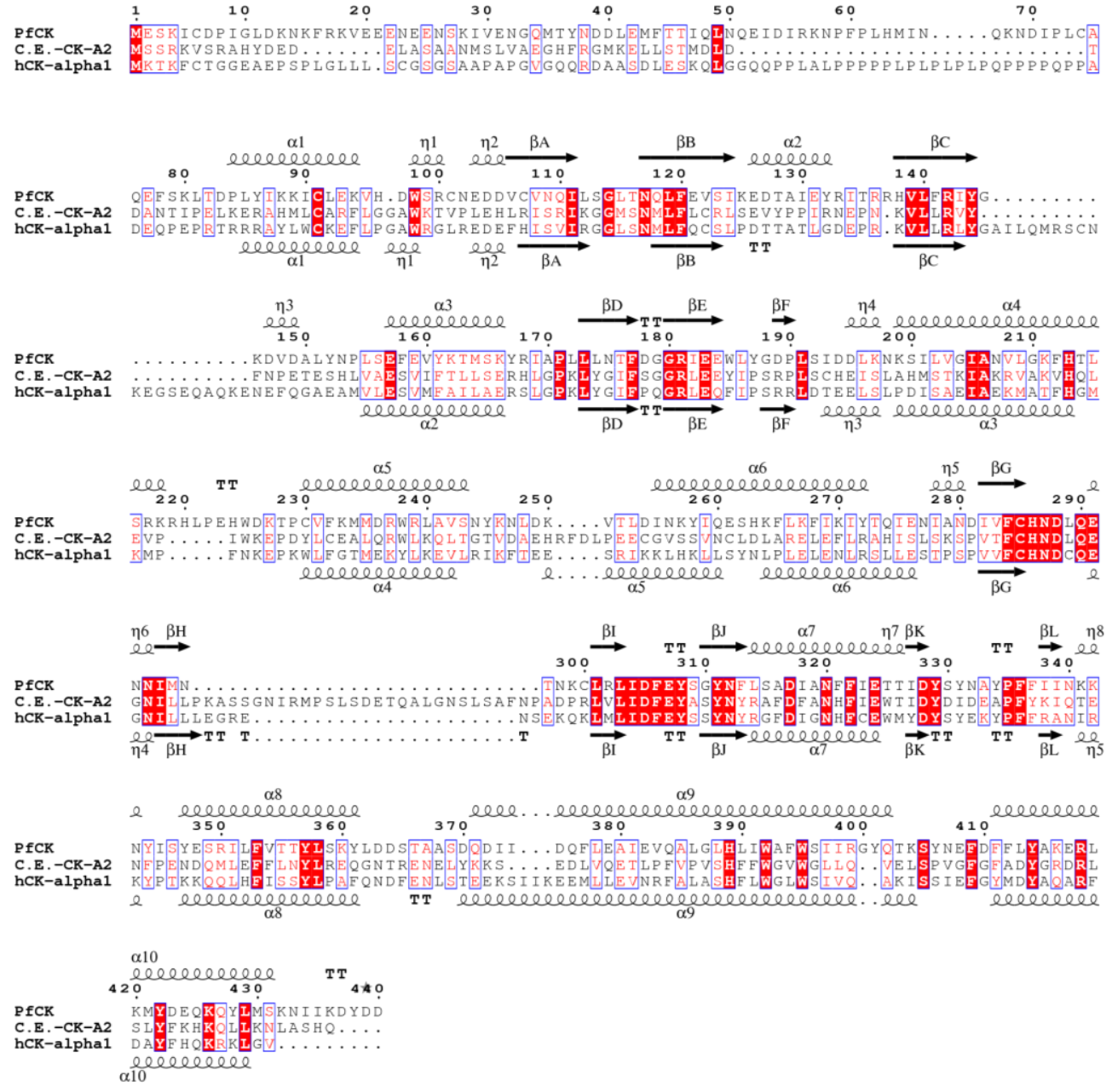

Figure 1. Sequence alignment of the full sequences of $P$. falciparum choline kinase (PfChoK), $C$. Elegans ChoK-A2 and hChoK $\alpha 1$. In the alignment, the secondary structure of the PfChoK structure is shown on the top, while the secondary structure of hChoK $\alpha 1$ is shown at the bottom. Helices $(\alpha)$ are indicated by numbers, while strands $(\beta)$ by alphabetical letters. PfChoK exhibits a sequence identity of $23.0 \%$ with ChoK-A2 and $25.3 \%$ with hChoK $\alpha 1$, respectively. Figure obtained with ESPript-http://espript.ibcp.fr [40].
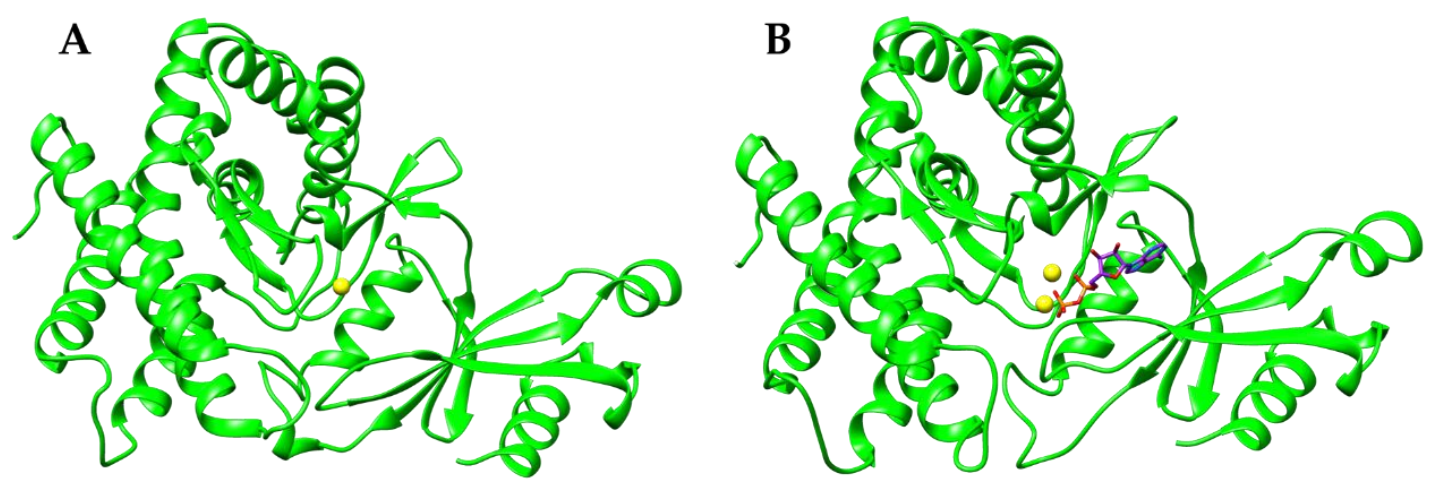

Figure 2. Overall fold of the PfChoK apoenzyme (PDB code: 6YXS) (A) and the ADP-bound PfChoK enzyme (PDB code: 6YXT) (B). Proteins are shown as green cartoons, while magnesium ions are depicted as yellow spheres and the ADP molecule in panel B is shown in sticks with carbon atoms in purple, nitrogen atoms in blue, phosphorus atoms in orange and oxygen atoms in red. 


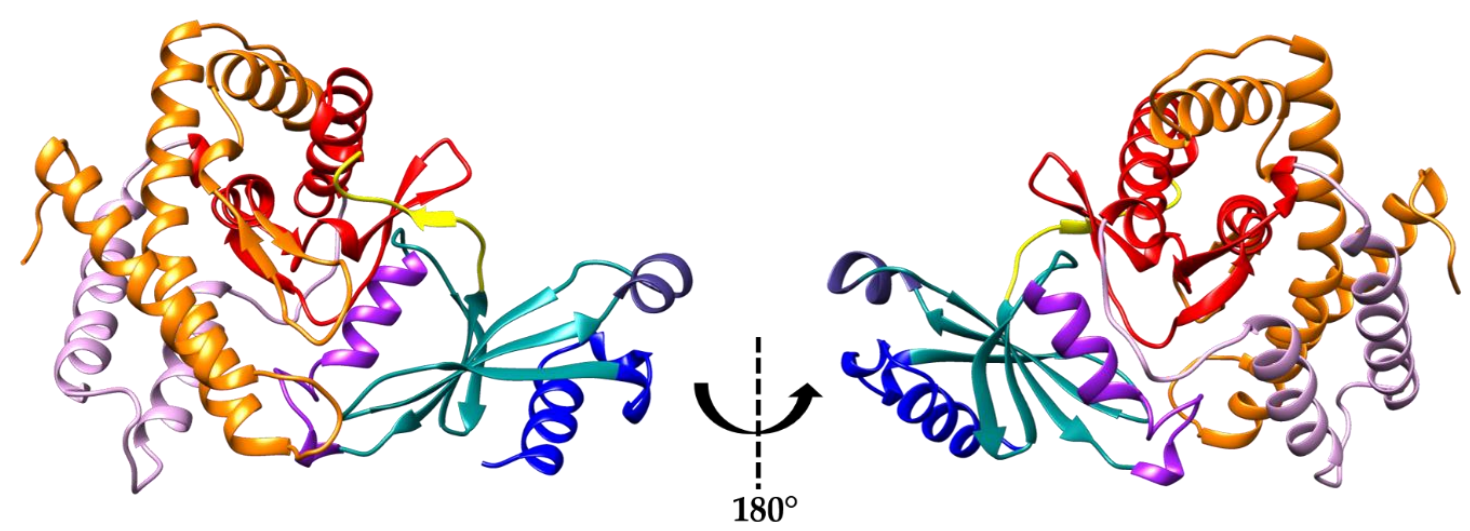

Figure 3. Two 180-degree rotated views of the PfChoK apoenzyme structure (PDB code: 6YXS). The atypical protein kinase fold regions are depicted in color code: helices $\alpha 1, \eta 1$, and $\eta 2$ are colored in blue; strands $\beta \mathrm{A}, \beta \mathrm{B}, \beta \mathrm{C}$, and $\beta \mathrm{D}$ in light sea green; helix $\alpha 2$ in purple, helices $\eta 3$, and $\alpha 3$ in magenta; $\beta$ strand $\mathrm{F}$ in yellow; the central core subdomain in red; the insertion subdomain in pink; and the C-terminal subdomain in orange.

The N-terminal domain is connected to the $\mathrm{C}$-terminal one by a short linker featuring a short $\beta$ strand ( $\beta F$, Figure 3, yellow) within a longer loop portion that makes a 90 degree turn. The PfChoK C-terminal domain is divided in three regions: the central core subdomain (Figure 3, red), the insertion subdomain (Figure 3, pink), and the C-terminal subdomain (Figure 3, orange). The central core subdomain is composed by helices $\eta 4, \alpha 4$, and $\alpha 7$ and by a harpin-shaped loop that contains two $\beta$ sheets (formed by the antiparallel strands $\beta \mathrm{G}, \beta \mathrm{H}$, and $\beta \mathrm{I}, \beta \mathrm{J}$ ) and $\eta 6$, a $3_{10}$ helix. These two $\beta$ sheets are especially relevant for PfChoK catalytic activity since they house the Brenner's motif ( $\beta \mathrm{G}, \beta \mathrm{H}$, and $\eta 6)$ and the choline kinase motif ( $\beta \mathrm{I}, \beta \mathrm{J}$ ).

The insertion subdomain is placed between helix $\alpha 4$ and strand $\beta G$ and is composed by two helices, $\alpha 5$ and $\alpha 6$, which are connected by a loop and by helix $\eta 5$. In the PfChoK structure, this insertion is located behind the central core and the $\mathrm{C}$-terminal subdomains and, unlike the $C$. Elegans and the human enzymes, where a long $\beta$ hairpin motif and an $\alpha$ helix are present between the helices corresponding to $\alpha 5$ and $\alpha 6$, it features a loop.

The C-terminal subdomain is located between the insertion and the core subdomains and it is connected to the final portion of the central core subdomain by a hairpin-like loop containing a $\beta$ sheet composed by $\beta$ strands $\mathrm{K}$ and L. Since this hairpin-like loop is positioned in front of the choline binding site, it is believed that it may regulate the accessibility to the active site; hence, the binding of choline, as proposed previously [38].

The harpin-like loop is followed by helices $\eta 8, \alpha 8, \alpha 9$, and $\alpha 10$, which compose the remaining of the C-terminal subdomain. Helix $\alpha 8$ is situated beneath helix $\alpha 4$ and is connected to helix $\alpha 9$ through a reverse turn. Helix $\alpha 9$ runs across the whole molecule and features a kink in its middle region. Another reverse kink connects helix $\alpha 9$ to $\alpha 10$, which extends towards the C-terminus of the enzyme. While structure superimposition between the apo and the ADP-bound form of PfChoK shows no significant conformational variability (r.m.s.d. $=0.38 \AA$ for $\mathrm{C} \alpha$ atoms), a comparison between the PfChoK apoenzyme and ChoKA-2 (PDB code: $1 \mathrm{NW} 1$ ) and hCK $\alpha 1$ (PDB code: $2 \mathrm{CKO}$ ) shows relatively high r.m.s.d. values ( $3.75 \AA$ and $2.35 \AA$, respectively, Supplementary Materials Figure S2). This is despite the overall good sequence alignment and the similar folding topology. However, the r.m.s.d. values get smaller when the individual $\mathrm{N}$-terminal domains are considered (1.6 ̊ for ChoKA- 2 and $1.15 \AA$ for hChoK $\alpha 1$ ), while they remain relatively high for the C-terminal domain ( $2.10 \AA$ for ChoKA-2 and $2.20 \AA$ for $h C h o K \alpha 1$ ). These r.m.s.d. values for the $\mathrm{C}$-terminal domain seem to be due to the marked differences in the secondary structure of the insertion subdomain. 
From a phylogenetic point of view, choline kinases have been classified as members of the atypical kinases (AK) family, a subgroup of eukaryotic protein kinases having a homologous catalytic core but not having all the other usual kinase motifs conserved [41].

Aside from the conserved catalytic central core domain, other AK family structural features are also present in the crystal structure of the PfChoK apoenzyme (Figure 4). For instance, two highly conserved residues, Arg142 and Glu183 form a salt bridge that plays an important stabilizing role in the architecture of the N-terminal domain (Figure 4A). Moreover, Arg142 has a role in the binding of ADP since it can interact with both its $\alpha$ and its $\beta$ phosphate group.
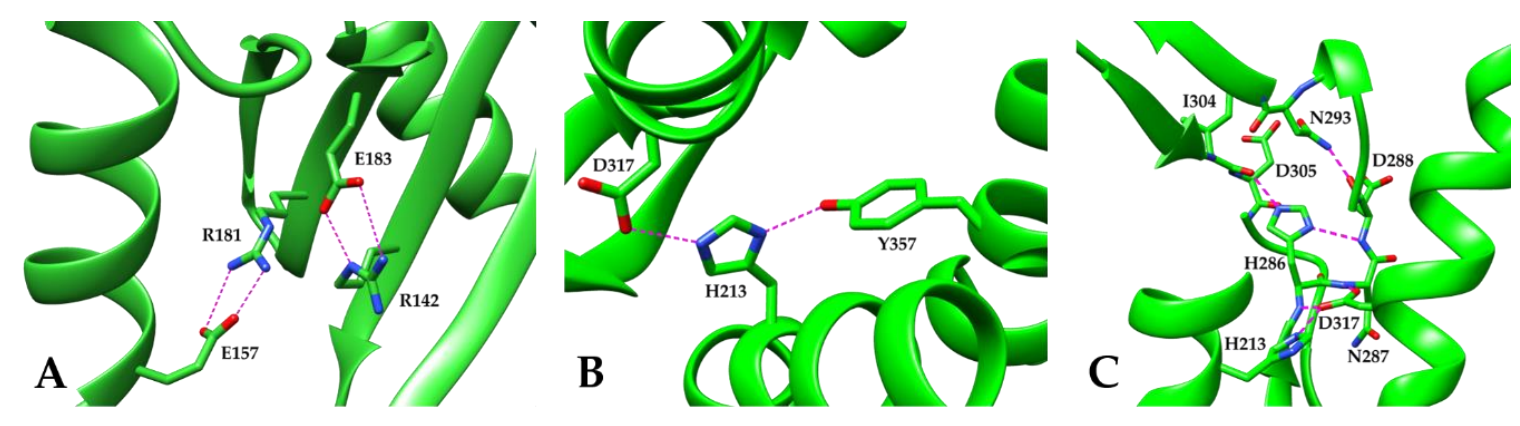

Figure 4. (A) The salt bridge interactions between Arg142 and Glu183 and Arg181 and Glu157 in the N-terminal domain. (B) Stabilizing role of His213, which forms H-bonding interactions with Asp317 and Tyr357. (C) hydrogen bonding network stabilizing the central catalytic core domain. The protein is shown as green cartoon, while highlighted residues are depicted in sticks colored with green carbon atoms, blue nitrogen atoms, and red oxygen atoms. Structural details are from the structure of the PfChoK apoenzyme (PDB code: 6YXS).

Another salt bridge between conserved residues in the ChoK family is formed between Arg181 and Glu157; thus, stabilizing the interaction between $\beta$ strand $\mathrm{E}$ and helix $\alpha 3$ (Figure 4A).

The residue His213 (Figure 4B), which is situated in helix $\alpha 4$, is also highly conserved and possesses an important structural role. Indeed, by linking together three different helices of the C-terminal domain, it greatly contributes to the overall fold stability. This linkage is provided by the side chain of His213 forming a hydrogen bond with the hydroxyl group of Tyr357 in helix $\alpha 8$ and with the carboxyl group of Asp317 in helix $\alpha 7$.

Another important structural motif that contributes to the stability of the eukaryotic protein kinase fold, and in particular of its catalytic central core, is the hydrogen-bonding network that involves those highly conserved residues that coordinate magnesium. This network (Figure 4C) features a hydrogen bond between His213 and Asp317, while the side chain of the latter contacts the nitrogen atoms of the backbone amide group of His286 and Asn287 situated in the Brenner's motif. The imidazole ring of His286 is sandwiched between the backbone carbonyl groups of Ile304 and Asp305 situated in the choline kinase motif and the backbone nitrogen of Asp288 positioned in the Brenner's motif. The backbone carbonyl group of Asp288 hydrogen bonds the nitrogen in the amide group of Asn293, which is located in helix $\eta 6$ in the Brenner's motif and whose side chain is also involved with the carbonyl group of Asp305 in the coordination of the catalytic magnesium. Four water molecules complete the octahedral coordination of the magnesium ion. All metal coordination distances are within a 2.02-2.15 Å range (Figure 5A). 


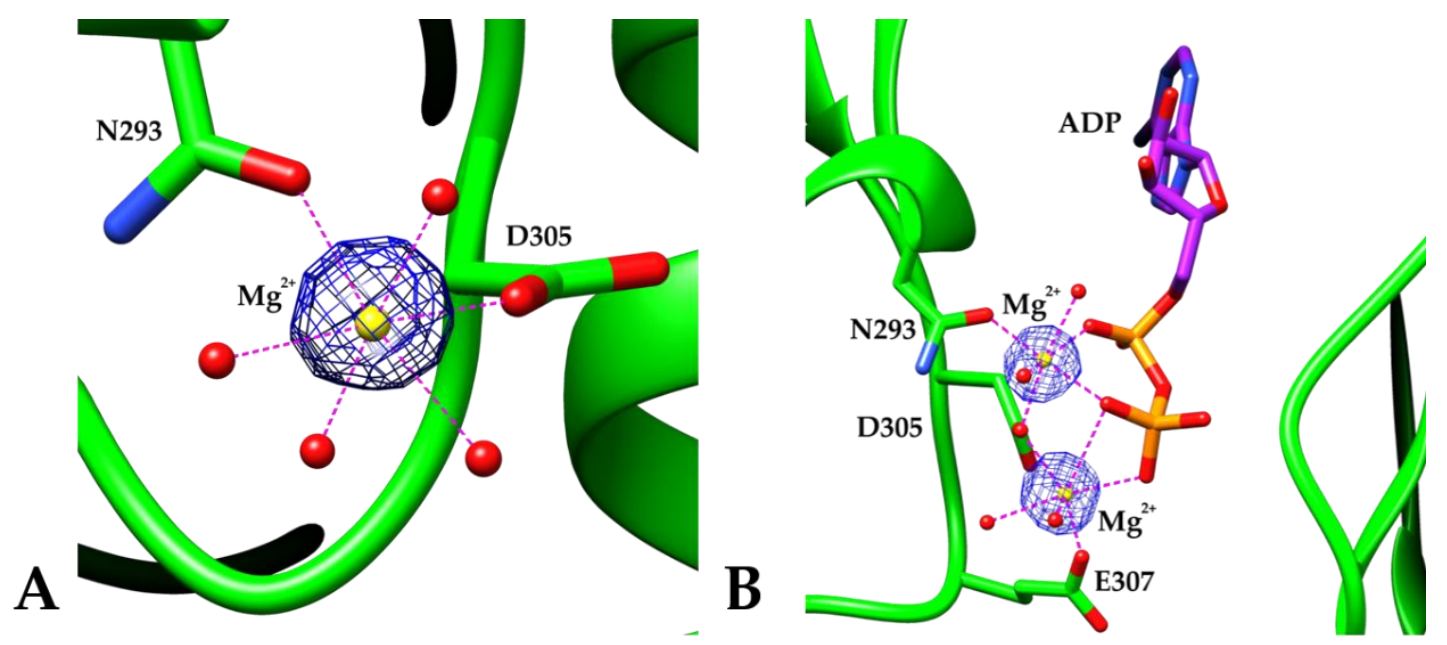

Figure 5. Magnesium ion coordination in the apoenzyme (PDB code: 6YXS) (A) and in the ADP-bound form (PDB code: 6YXT) (B). Magnesium ions are depicted in small yellow spheres, interacting residues are indicated with the corresponding number and one letter code, shown in ball and stick and are colored with carbon atoms in green, nitrogen atoms in blue, and oxygen atoms in red. The electron density from omit maps is contoured at the $4.0 \sigma$ level. The ADP molecule is drawn in stick with purple carbon atoms, red oxygen atoms and orange phosphorus atoms.

The ADP-bound structure reveals the binding mode of the adenosine nucleotide in PfChoK (Figure 6). The ligand is found in a hydrophobic pocket comprised by the N-terminal domain and the C-terminal central core domain and involves many residues of the Brenner's motif. The hydrophobic pocket is mainly lined by non-polar residues, such as Ile111, Leu112, Ser113, Leu140, Pro171, Trp185, Leu186, Pro190, Met295, and Ile304. Moreover, ADP is further stabilized within the enzyme by a number of hydrogen bonds. In particular, the backbone amide group of Gly114 interacts with one of the oxygen atoms in the $\beta$ phosphate group ( $2.87 \AA$ ), the side chain of Asn 117 makes a hydrogen bond with the same oxygen of the $\beta$ phosphate group ( $2.98 \AA$ ) and the backbone carbonyl group of Glu184 hydrogen bonds the nitrogen in the adenine ring ( $2.84 \AA$ ). Furthermore, here the highly conserved Arg142 is observed in the proximity of one of the oxygen atoms of the $\alpha$ phosphate group of ADP at a distance of $3.42 \AA$, forming an electrostatic interaction.

The ADP molecule also participates in the coordination of two magnesium ions (Figure 5B). Unlike the regular octahedral coordination of the magnesium ion in the apoenzyme structure, here the octahedral coordination is highly distorted due to the participation of the ADP phosphate groups in the metal coordination sphere. One magnesium ion is coordinated by the side chains of Asp305 and Glu307, by two water molecules and by two oxygen atoms of the $\beta$ phosphate group, while the second one is coordinated by the side chains of Asn293 and Asp305, two water molecules and two oxygen atoms of the ADP molecule originating from the $\alpha$ and $\beta$ phosphate groups.

In our PfChoK structure (6YXT), ADP binds to the same ADP-binding motif as in hChoK $\alpha 1$ (2CKP) [39], in the incomplete ADP- and phosphocholine-bound form of the P. falciparum enzyme (3FI8, unpublished data), and in the co-crystal structure of hChoK $\alpha 1$, ADP, and hemicholinium-3 (3G15) [42] (Figure 7), even though, in all these structures, the presence and the number of magnesium ions vary. 

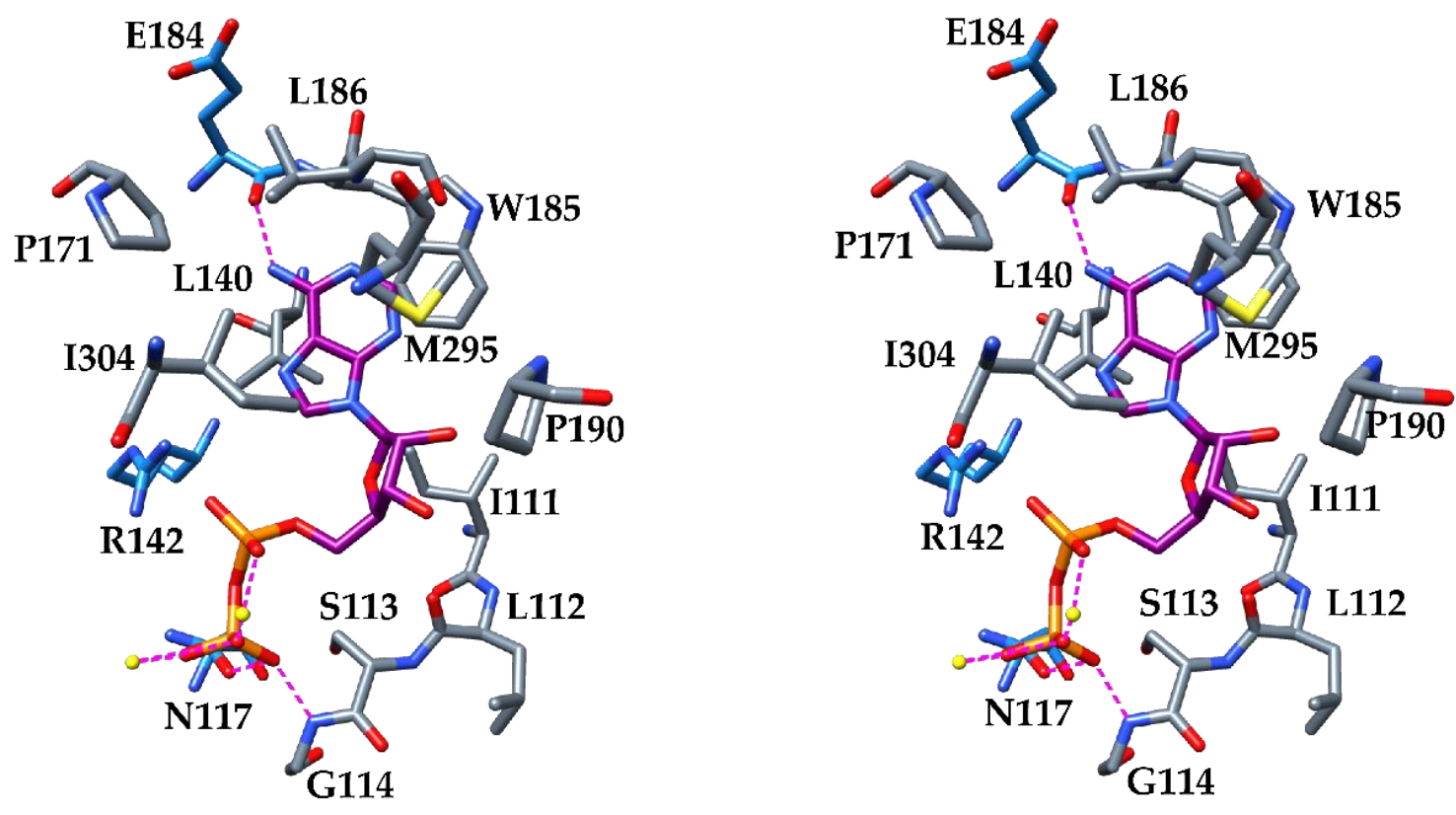

Figure 6. Stereodiagram of the ADP binding mode. The hydrophobic interactions with the non-polar residues that line the pocket in which ADP is bound are shown with grey carbon atoms, while the polar residues that interact with ADP by hydrogen bonding are shown with light blue carbon atoms. All protein residues are drawn in ball and stick, while the ADP molecule is drawn in sticks and shown with purple carbons. Nitrogen atoms are colored in blue, oxygen atoms in red, and phosphor atoms in orange. Structural details are from the ADP-bound form of PfChoK (PDB code: 6YXT).

A

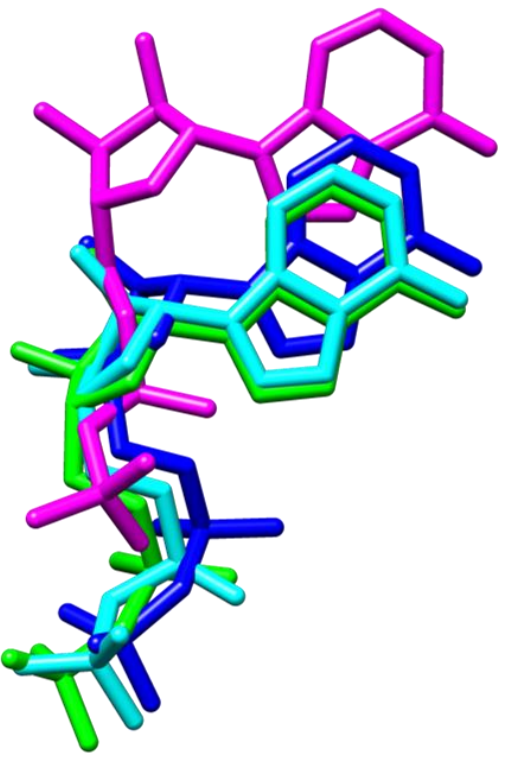

B

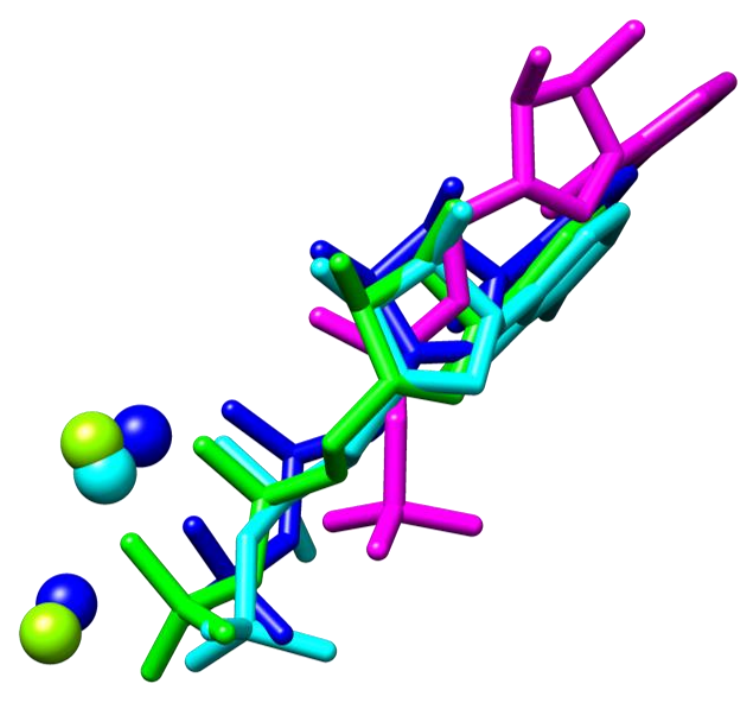

Figure 7. (A) Structural superimposition of ADP molecules in the ADP-bound form of PfChoK (PDB code: 6YXT, green), the ADP- and phosphocholine-bound form of PfChoK (PDB code: 3FI8, cyan), the ADP-bound form of hChoK $\alpha 1$ (PDB code: 2 CKP, magenta), and the co-crystal structure of hChoK $\alpha 1$, ADP and hemicholinium-3 (PDB code: 3G15, blue). (B) Structural superimposition of ADP and magnesium ions with the same models and with the same color code. ADP molecules are shown in sticks and magnesium ions are drawn as spheres.

By comparing our ADP-bound PfChoK structure (6YXT) with 3FI8, we cannot detect any differences in the enzyme portion and in the residues that bind the ADP molecule, the only appreciable difference between the two structures being the presence of only one magnesium ion that is coordinated by 
Asn293 and Asp305 (cyan sphere in Figure 7B) and that interacts with ADP. The other magnesium ion that is present in 6YXT is not found in 3FI8. The positions of the magnesium ions and of ADP in our PfChoK structure (6YXT) are similar to those observed in the ADP- and hemicholinium-3-bound $\mathrm{hChoK} \alpha 1$ structure (3G15). Since it is known that two magnesium ions are required to perform the breaking of the ATP phosphodiester bond, the varying number of magnesium ions that are present in the crystal structures may suggest that these are different stages in the catalytic process that leads to the production of phosphocholine.

As for the choline binding site, the majority of the residues that bind choline in the human enzyme are conserved in PfChoK as well (Figure 8). In the hChoK $\alpha 1$, the choline binding site is an essentially hydrophobic pocket that is capped by negatively charged residues [39]. Hence, two different functional regions can be identified: one lined by Asp215, Glu217, Glu218, Glu309, Glu349, Asp353, Glu357, and Glu434 (hChoK $\alpha 1$ numbering, Figure 8), which are involved in the electrostatic steering of choline and in the binding of the quaternary amine; the other forming a groove lined by Tyr333, Tyr354, Trp420, Trp423, and Tyr440, which provide direct binding to choline. It should be noted that there are no structural differences between the human apoenzyme (2CKO) and the human phosphocholine-bound form of the enzyme (2CKQ) (Figure 8, orange, and magenta residues).

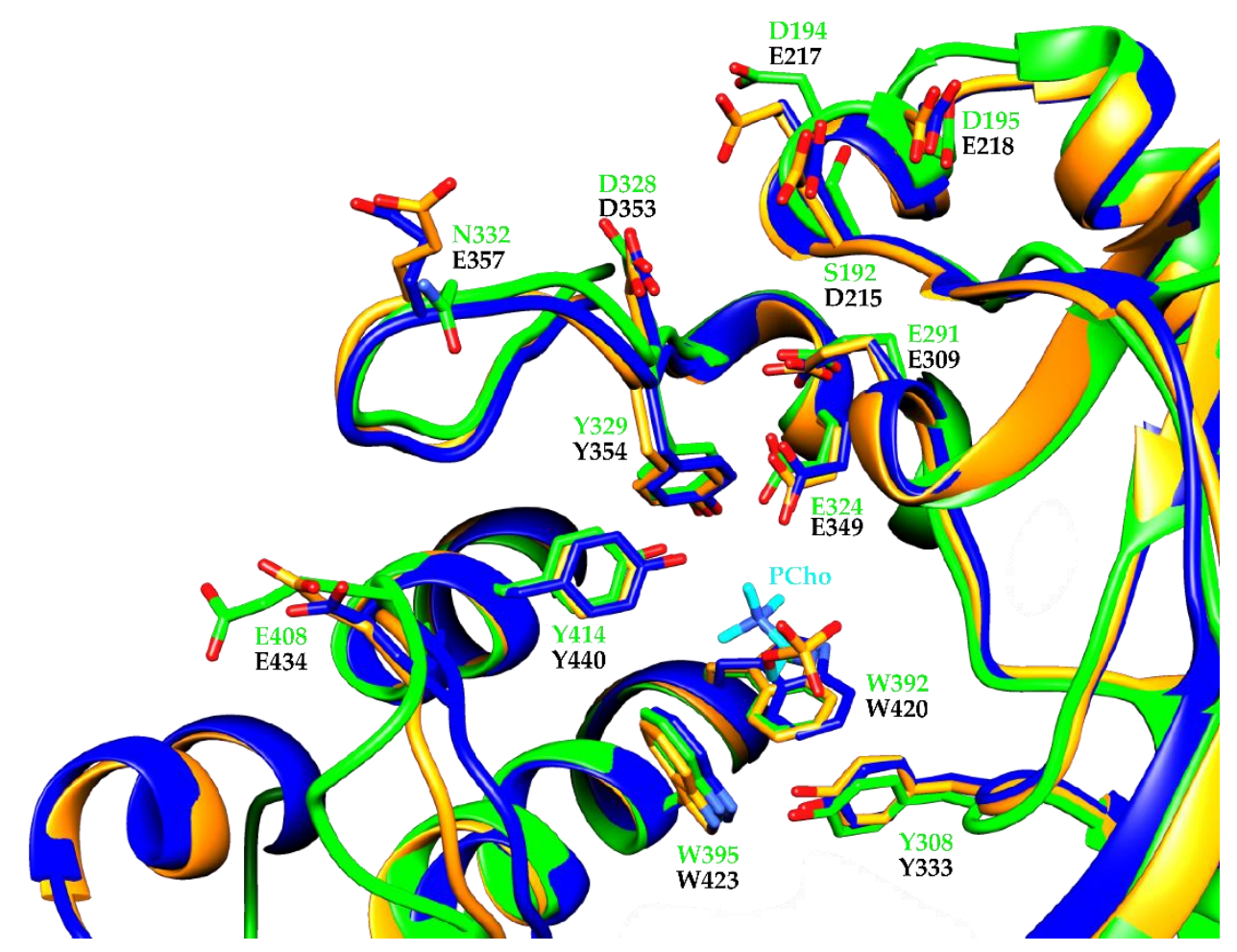

Figure 8. Structure alignment of the PfChoK (green cartoon) and the hChoK $\alpha 1$ (blue and orange cartoons) choline binding sites. The alignment was obtained by superimposing the human apoenzyme (PDB code: $2 \mathrm{CKO}$, orange cartoon), the phosphocholine-bound form of the human enzyme (PDB code: 2CKQ, blue cartoon) and the apo form of PfChoK (PDB code: 6XYS, green cartoon). Residues involved in choline binding are shown in sticks with orange, blue or green carbon atoms, blue nitrogen atoms, and red oxygen atoms. Residues are labeled with the corresponding one letter code and residue number, which is green for PfChoK and black for 2CKO and 2CKQ. Phosphocholine (labelled PCho) is shown in sticks with carbon atoms in cyan, nitrogen atoms in blue, oxygen atoms in red, and phosphorous atoms in yellow.

In PfChoK, while the hydrophobic residues lining the choline-binding pocket are conserved (Tyr308, Tyr329, Trp392, Trp395, and Tyr414 in the parasite enzyme sequence) and superimpose well with the corresponding residues in the choline-binding site of the human enzyme, the majority of the 
differences are among the negatively charged residues involved in choline binding. Indeed, Glu217, Glu218, and Glu357 in the human enzyme are replaced by Asp194, Asp195, and Asn332 in PfChoK, the latter of the three substitutions altering the electrostatic landscape of the loop that is found in close proximity to the pocket. Finally, Asp 215 of hChoK $\alpha 1$ is replaced by Ser192 in PfChoK. All other residues (Glu309, Glu349, and Asp353) in hChoK $\alpha 1$ are conserved and superimpose well with their PfChoK counterparts (Glu291, Glu324, and Asp328).

\section{Conclusions}

To date, despite its relevance as a drug target against malaria, PfChoK has been only partially characterized from a structural viewpoint. As a result, the three-dimensional arrangement of the corresponding C. elegans and human enzyme have often served for homology modeling purposes. Here, we reported the complete structural characterization of the apo and the ADP-bound form of the enzyme; thus, shedding light on previously elusive features of the PfChoK structure and catalytic cycle and facilitating a structure-based drug discovery approach to this important target.

Supplementary Materials: The following are available online at http://www.mdpi.com/2073-4352/10/7/613/s1, Figure S1: Analytical size-exclusion chromatography, Figure S2: Structural superposition of PfChoK (6YXS), CHA-2 (1NW1) and ChoK $\alpha 1$ (2CKO), Figure S3: omit map of the ADP molecule (6YXT).

Author Contributions: Conceptualization, L.C.L.-C. and E.P.; methodology, A.T. and E.P.; validation, A.T. and E.P.; investigation, A.T. and E.P.; writing — original draft preparation, A.T. and E.P.; writing—review and editing, A.T., L.C.L.-C., and E.P.; supervision, E.P.; and project administration, E.P. All authors have read and agreed to the published version of the manuscript.

Funding: This research received no external funding.

Acknowledgments: The authors thank the X06DA-PXIII beamline personnel of the Paul Scherrer Institute, Villigen, Switzerland, for help with data collection. Atomic coordinates and structure factors for the reported crystal structures have been deposited in the Protein Data Bank under the accession codes 6YXS and 6YXT.

Conflicts of Interest: The authors declare no conflict of interest.

\section{References}

1. World Health Organization. World Malaria Report 2019; World Health Organization: Geneva, Switzerland, 2019; ISBN 9789241565721.

2. Mitamura, T.; Marie, N.; Palacpac, Q. Lipid metabolism in Plasmodium falciparum-infected erythrocytes: Possible new targets for malaria chemotherapy. Microbes Infect. 2003, 5, 545-552. [CrossRef]

3. World Health Organization. Guidelines for the Treatment of Malaria; World Health Organization: Geneva, Switzerland, 2015.

4. Su, X.Z.; Lane, K.D.; Xia, L.; Sá, J.M.; Wellems, T.E. Plasmodium genomics and genetics: New insights into malaria pathogenesis, drug resistance, epidemiology, and evolution. Clin. Microbiol. Rev. 2019, 32, 1-29. [CrossRef]

5. Blasco, B.; Leroy, D.; Fidock, D.A. Antimalarial drug resistance: Linking Plasmodium falciparum parasite biology to the clinic. Nat. Med. 2017, 23, 917-928. [CrossRef] [PubMed]

6. Krishna, S. Science, medicine, and the future. Malar. BMJ 1997, 325, 730-732.

7. Trampuz, A.; Jereb, M.; Muzlovic, I.; Prabhu, R.M. Clinical review: Severe malaria. Crit. Care 2003, 7, 315-323. [CrossRef]

8. Taraschi, T.F.; Parashar, A.; Hooks, M.; Rubin, H. Perturbation of red cell membrane structure during intracellular maturation of Plasmodium falciparum. Science 1986, 232, 102-104. [CrossRef] [PubMed]

9. Simões, A.P.; Moll, G.N.; Slotboom, A.J.; Roelofsen, B.; Op den Kamp, J.A.F. Selective internalization of choline-phospholipids in Plasmodium falciparum parasitized human erythrocytes. BBA-Biomembranes 1991, 1063, 45-50.

10. Ancelin, M.L.; Vial, H.J.; Calas, M.; Giral, L.; Piquet, G.; Rubi, E.; Thomas, A.; Peters, W.; Slomianny, C.; Herrera, S. Present development concerning antimalarial activity of phospholipid metabolism inhibitors with special reference to in vivo activity. Mem. Inst. Oswaldo Cruz 1994, 89 (Suppl. 2), 85-90. [CrossRef] 
11. Calas, M.; Cordina, G.; Bompart, J.; Bari, M.B.; Jei, T.; Ancelin, M.L.; Vial, H. Antimalarial activity of molecules interfering with plasmodium falciparum phospholipid metabolism. Structure-activity relationship analysis. J. Med. Chem. 1997, 2623, 3557-3566. [CrossRef]

12. Ancelin, B.M.L.; Calas, M.; Bompart, J.; Cordina, G.; Martin, D.; Bari, M.B.; Jei, T.; Druilhe, P.; Vial, H.J. Antimalarial activity of 77 phospholipid polar head analogs: Close correlation between inhibition of phospholipid metabolism and in vitro plasmodium falciparum growth. Blood 1998, 91, 1426-1437. [CrossRef]

13. Ancelin, M.L.; Calas, M.; Vidal-Sailhan, V.; Herbuté, S.; Ringwald, P.; Vial, H.J. Potent inhibitors of Plasmodium phospholipid metabolism with a broad spectrum of in vitro antimalarial activities. Antimicrob. Agents Chemother. 2003, 47, 2590-2597. [CrossRef]

14. Kilian, N.; Choi, J.Y.; Voelker, D.R.; Mamoun, C. Ben Role of phospholipid synthesis in the development and differentiation of malaria parasites in the blood. J. Biol. Chem. 2018, 293, 17308-17316. [CrossRef] [PubMed]

15. Vial, H.J.; Ancelin, M.L. Malarial lipids. In Malaria: Parasite Biology, Phatogenesis, and Protection; Sherman, I.W., Ed.; American Society for Microbiology Press: Washington, DC, USA, 1998; pp. 159-175.

16. Ancelin, M.L.; Vial, H.J. Choline kinase activity in Plasmodium-infected erythrocytes: Characterization and utilization as a parasite-specific marker in malarial fractionation studies. Biochim. Biophys. Acta (BBA)/Lipids Lipid Metab. 1986, 875, 52-58. [CrossRef]

17. Ancelin, M.L.; Vial, H.J. Quaternary ammonium compounds efficiently inhibit Plasmodium falciparum growth in vitro by impairment of choline transport. Antimicrob. Agents Chemother. 1986, 29, 814-820. [CrossRef] [PubMed]

18. Salom-Roig, X.; Hamze, A.; Calas, M.; Vial, H. Dual molecules as new antimalarials. Comb. Chem. High Throughput Screen. 2005, 8, 49-62. [CrossRef]

19. Peyrottes, S.; Caldarelli, S.; Wein, S.; Périgaud, C.; Pellet, A.; Vial, H. Choline analogues in malaria chemotherapy. Curr. Pharm. Des. 2012, 18, 3454-3466. [CrossRef]

20. Pessi, G.; Choi, J.Y.; Reynolds, J.M.; Voelker, D.R.; Ben Mamoun, C. In vivo evidence for the specificity of Plasmodium falciparum phosphoethanolamine methyltransferase and its coupling to the Kennedy Pathway. J. Biol. Chem. 2005, 280, 12461-12466. [CrossRef]

21. Déchamps, S.; Shastri, S.; Wengelnik, K.; Vial, H.J. Glycerophospholipid acquisition in Plasmodium-A puzzling assembly of biosynthetic pathways. Int. J. Parasitol. 2010, 40, 1347-1365. [CrossRef]

22. Wein, S.; Ghezal, S.; Buré, C.; Maynadier, M.; Périgaud, C.; Vial, H.J.; Lefebvre-Tournier, I.; Wengelnik, K.; Cerdan, R. Contribution of the precursors and interplay of the pathways in the phospholipid metabolism of the malaria parasite. J. Lipid Res. 2018, 59, 1461-1471. [CrossRef]

23. Gibellini, F.; Smith, T.K. The Kennedy pathway-de novo synthesis of phosphatidylethanolamine and phosphatidylcholine. IUBMB Life 2010, 62, 414-428. [CrossRef]

24. Choubey, V.; Maity, P.; Guha, M.; Kumar, S.; Srivastava, K.; Puri, S.K.; Bandyopadhyay, U. Inhibition of Plasmodium falciparum choline kinase by hexadecyltrimethylammonium bromide: A possible antimalarial mechanism. Antimicrob. Agents Chemother. 2007, 51, 696-706. [CrossRef] [PubMed]

25. Zimmerman, T.; Moneriz, C.; Diez, A.; Bautista, J.M.; Del Pulgar, T.G.; Cebrián, A.; Lacal, J.C. Antiplasmodial activity and mechanism of action of RSM-932A, a promising synergistic inhibitor of Plasmodium falciparum choline kinase. Antimicrob. Agents Chemother. 2013, 57, 5878-5888. [CrossRef]

26. Serran-Aguilera, L.; Nuti, R.; Lopez-Cara, L.C.; Rios-Marco, P.; Carrasco, M.P.; Marco, C.; Entrena, A.; Macchiarulo, A.; Hurtado-Guerrero, R. Choline kinase active site provides features for designing versatile inhibitors. Curr. Top. Med. Chem. 2014, 14, 2684-2693. [CrossRef] [PubMed]

27. Serrán-Aguilera, L.; Denton, H.; Rubio-Ruiz, B.; López-Gutiérrez, B.; Entrena, A.; Izquierdo, L.; Smith, T.K.; Conejo-García, A.; Hurtado-Guerrero, R. Plasmodium falciparum choline kinase inhibition leads to a major decrease in phosphatidylethanolamine causing parasite death. Sci. Rep. 2016, 6, 1-12. [CrossRef] [PubMed]

28. Schiafino-Ortega, S.; Baglioni, E.; Pérez-Moreno, G.; Marco, P.R.; Marco, C.; González-Pacanowska, D.; Ruiz-Pérez, L.M.; Carrasco-Jiménez, M.P.; López-Cara, L.C. 1,2-Diphenoxiethane salts as potent antiplasmodial agents. Bioorg. Med. Chem. Lett. 2018, 28, 2485-2489. [CrossRef]

29. Ancelin, M.L.; Vial, H.J. Several lines of evidence demonstrating that Plasmodium falciparum, a parasitic organism, has distinct enzymes for the phosphorylation of choline and ethanolamine. FEBS Lett. 1986, 202, 217-223. [CrossRef] 
30. Choubey, V.; Guha, M.; Maity, P.; Kumar, S.; Raghunandan, R.; Maulik, P.R.; Mitra, K.; Halder, U.C.; Bandyopadhyay, U. Molecular characterization and localization of Plasmodium falciparum choline kinase. Biochim. Biophys. Acta-Gen. Subj. 2006, 1760, 1027-1038. [CrossRef]

31. Alberge, B.; Gannoun-Zaki, L.; Bascunana, C.; Tran Van Ba, C.; Vial, H.; Cerdan, R. Comparison of the cellular and biochemical properties of Plasmodium falciparum choline and ethanolamine kinases. Biochem. J. 2010, 425, 149-158. [CrossRef]

32. Battye, T.G.G.; Kontogiannis, L.; Johnson, O.; Powell, H.R.; Leslie, A.G.W. iMOSFLM: A new graphical interface for diffraction-image processing with MOSFLM. Acta Crystallogr. Sect. D 2011, 67, 271-281. [CrossRef] [PubMed]

33. Winn, M.D.; Ballard, C.C.; Cowtan, K.D.; Dodson, E.J.; Emsley, P.; Evans, P.R.; Keegan, R.M.; Krissinel, E.B.; Leslie, A.G.W.; McCoy, A.; et al. Overview of the CCP4 suite and current developments. Acta Crystallogr. Sect. D 2011, 67, 235-242. [CrossRef]

34. Liebschner, D.; Afonine, P.V.; Baker, M.L.; Bunkóczi, G.; Chen, V.B.; Croll, T.I.; Hintze, B.; Hung, L.-W.; Jain, S.; McCoy, A.J.; et al. Macromolecular structure determination using X-rays, neutrons and electrons: Recent developments in Phenix. Acta Crystallogr. Sect. D 2019, 75, 861-877. [CrossRef]

35. Emsley, P.; Lohkamp, B.; Scott, W.G.; Cowtan, K. Features and development of Coot. Acta Crystallogr. Sect. D Biol. Crystallogr. 2010, 66, 486-501. [CrossRef] [PubMed]

36. Pettersen, E.F.; Goddard, T.D.; Huang, C.C.; Couch, G.S.; Greenblatt, D.M.; Meng, E.C.; Ferrin, T.E. UCSF Chimera-A visualization system for exploratory research and analysis. J. Comput. Chem. 2004, 25, 1605-1612. [CrossRef] [PubMed]

37. Delano, W.L. The PyMOL Molecular Graphics System, Version 1.8; Schrödinger LLC: New York, NY, USA, 2002; Volume 10.

38. Peisach, D.; Gee, P.; Kent, C.; Xu, Z. The crystal structure of choline kinase reveals a eukaryotic protein kinase fold. Structure 2003, 11, 703-713. [CrossRef]

39. Malito, E.; Sekulic, N.; Cun, W.; Too, S.; Konrad, M.; Lavie, A. Elucidation of human choline kinase crystal structures in complex with the products ADP or phosphocholine. J. Mol. Biol. 2006, 364, 136-151. [CrossRef]

40. Robert, X.; Gouet, P. Deciphering key features in protein structures with the new ENDscript server. Nucleic Acids Res. 2014, 42, W320-W324. [CrossRef]

41. Scheeff, E.D.; Bourne, P.E. Structural evolution of the protein kinase-like superfamily. PLoS Comput. Biol. 2005, 1, e49. [CrossRef]

42. Hong, B.S.; Allali-Hassani, A.; Tempel, W.; Finerty, P.J.; MacKenzie, F.; Dimov, S.; Vedadi, M.; Park, H.W. Crystal structures of human choline kinase isoforms in complex with hemicholinium-3: Single amino acid near the active site influences inhibitor sensitivity. J. Biol. Chem. 2010, 285, 16330-16340. [CrossRef] 